\title{
Fuzzy Clustered Multi Objective Differential Evolution for Thermal Generator Maintenance Scheduling
}

\author{
G. Balaji ${ }^{1 *}$, R. Balamurugan ${ }^{1}$, L. Lakshminarasimman ${ }^{1}$ \\ ${ }^{1}$ Department of Electrical Engineering, Annamalai University \\ Annamalainagar, Tamilnadu, India \\ *Corresponding author's Email: balaji.g.au@gmail.com
}

\begin{abstract}
Generator Maintenance Scheduling (GMS) problem in a vertically integrated electric utility system is considered in this paper. The cost and reliability objectives are included in the problem formulation. The GMS is formulated as a non linear, heavily constrained multi-objective optimization problem and the non dominated solutions are obtained using multi objective hybrid integer coded differential evolution (MOHDE) algorithm. To attain the best compromise non dominated solution between cost and reliability, fuzzy based mechanism has been used in MOHDE. In this paper, fuzzy clustered multi objective hybrid differential evolution (FC-MOHDE) is proposed for solving thermal generator maintenance scheduling. To validate the performance of the proposed algorithm, two systems having 4 and 22 generating units are considered. The test results confirm the superiority of the algorithm in determining the best compromise solution for the multi objective GMS problem.
\end{abstract}

Keywords: Thermal generator maintenance scheduling; Multi objective hybrid differential evolution; Fuzzy mechanism; Best compromise solution; Optimal maintenance schedule.

\section{Introduction}

Since the environment is getting worse, the attitude of opposing erection of new thermal power plants spreads around the world. This leads to tight spinning reserve. Due to the increasing electric power demand, modern power systems operate with lower reserve margin. Under these circumstances, the maintenance of power production units plays a vital role in power system operation and planning problems that includes unit commitment, hydrothermal coordination and economic dispatch. Generally, long term generator maintenance scheduling is done for a planning horizon of one or two year. The prime objective of GMS is to find the precise time table for preventive maintenance of power generating units in order to minimize the operating cost, maximize the system reliability and to extend the life time of thermal generating units. The complexity of the problem increases with increase in system size. Over the past several decades, wide varieties of researches have been made towards GMS. In earlier works, heuristic methods and mathematical approaches are used for solving GMS. Heuristic methods schedule the generators for maintenance one after the other based on some predefined priority. However, this method fails to find even near optimal solution for GMS [1]. The increase in size of the system exponentially increases the intermediate solution space known as curse of dimensionality. Due to the curse of dimensionality, the mathematical approaches are limited only for solving small size system. To find solution for GMS of large scale systems in a reasonable amount of time, intelligent optimization techniques have been proposed. The objective of minimizing the cost is considered and schedule is obtained for maintenance of generators using combination of logic programming, constraint satisfaction technique and Branch and Bound (B \& B) algorithm [1], duality theory [2], Simulated Annealing (SA) [3], Genetic Algorithm (GA) [4], Tabu Search (TS) [5], Memetic Algorithm (MA) [6], Evolutionary Programming (EP) [7] and Particle Swarm Optimization (PSO) [8]. The reliability 
objective of GMS is as important as cost objective. The reliability associated with a power system is a measure of its capability to provide an adequate supply of electrical energy for the period of time. The reliability objective may be either deterministic or stochastic. The deterministic reliability criterion of level the reserve is considered as objective and schedule for maintenance of generators is obtained using TS [5]. The goal of minimizing sum of squares of reserve is taken as objective in GMS formulation and has been solved using novel GA in [9] and Ant Colony Optimization in [10]. Maintenance schedule is obtained using code specific and constraint-transparent integral coded GA in [11] based on the reliability objective of level the reserve rate. Several stochastic reliability criterions like loss of load expectation (LOLE), loss of load probability (LOLP) are taken as objective in formulating GMS and timetable for maintenance of power production units has been obtained using GA and method of cumulants in [12] and [13] respectively. Recently Harmony Search (HS) algorithm [14] and Teaching Learning based Optimization (TLBO) [15] have also been tried for the solution of GMS. In [16], Kim et al. have considered two objectives cost and reliability and converted into single objective with the help of weighting coefficients and maintenance timetable is obtained using hybrid GA, SA and TS. Leou [17] proposed a new formulation in which the cost and reliability are considered as an index and GA is combined with SA and is implemented for solving the problem. Multi objective formulation of GMS model is considered in [18] and non inferior solution for GMS is attained. Multi objective GMS model in which four objectives such as loss of load expectation, expected un-served energy, expected fuel cost and constraint violation are considered and has been solved using B \& B algorithm [19]. From the literature it can be seen that, only few works model the GMS as multi objective problem (MOP). This encourages the authors to consider and formulate GMS as nonlinear, constrained MOP. The GMS is a mixed integer problem that has both binary integer and continuous variables. The binary integers denote the on/off status of generators and continuous variables indicate the power output from the committed generating units.

Differential Evolution (DE) introduced by Storn and Price $[20,21]$ is a powerful population based evolutionary algorithm that shows outstanding performance in wide variety of problems in various fields of applications [22]. The conventional DE has the problem of premature convergence [22]. In order to avoid premature convergence and to find global optimal or near global optimal solution for a particular problem at hand, DE requires more population which leads to huge computational efforts. The problem like GMS has more number of decision variables. To obtain a global optimum value for such high dimension problems, DE needs large number of population. This also encourages the author to propose an algorithm for solving high dimensional GMS problem with considerable reduction in population size. In this paper, starting period for maintenance of power generating units is considered as decision variables which are integers. Thus the number of variables to be optimized is considerably reduced. DE is suitably modified to handle the integer variables. Lagrange multiplier method is an iterative mathematical method that is used to economically dispatch the available generation in order to meet the active power load demand with minimum production cost is also included into integer coded differential evolution (HDE) procedure. In order to get the non inferior solution set for multi objective GMS, in this paper, multi objective hybrid differential evolution (MOHDE) algorithm is proposed. In addition, to assist decision maker in identifying the best compromise non inferior solution, fuzzy set theory has been used and is incorporated into the MOHDE procedure. The major advantage of using this fuzzy clustering based algorithm is that it prunes away the use of an external memory that is used to store non dominated solution. Thus the proposed fuzzy clustered multi objective hybrid differential evolution (FC-MOHDE) algorithm finds the Pareto front and best compromise solution for GMS problem. To validate the proposed method for solving multi objective GMS, two case studies are considered.

This paper is organized as follows: Section 2 describes multi objective problem formulation of the thermal generator maintenance scheduling problem; Section 3 presents the proposed methodology for solving GMS; the simulation results and discussions of using the proposed technique to solve thermal GMS for 4 - and 22- units system are included in Sections 4; Section 5 presents the conclusions.

\section{Problem Formulation}

In this section, the various objectives related to generator maintenance scheduling problem subject to a set of equality and inequality constraints are described. The objectives considered here are (i) minimizing the overall operational cost and (ii) maximizing the deterministic reliability of the power system. If the overall operational cost is minimized, 
the system reliability is not that much good whereas if the reliability of the system is maximized, the overall operational cost also gets increased drastically. Hence in this paper, the GMS problem is formulated as a multi objective problem (MOP) in order to find a best compromise solution between cost and reliability. Here, the starting period for maintenance of power generating units is taken as decision variables. The GMS problem is formulated with the assumption that the power demand for each sub-period is known. The sub-period applied is normally one week.

\section{Minimizing the overall operational cost}

The overall operational cost is the sum of production cost and variable operation and maintenance cost. The objective function of minimizing the overall operational cost over the planning period is stated as

$F_{1}=\sum_{t=1}^{T} \sum_{i=1}^{N} H \cdot\left(a_{i}+b_{i} P_{i t}+c_{i} P_{i t}{ }^{2}\right) \cdot\left(1-U_{i t}\right)+\sum_{t=1}^{T} \sum_{i=1}^{N} H \cdot P_{i t} \cdot v_{i} \cdot\left(1-U_{i t}\right)(1)$

The first part of equation (1) is the overall operational cost and second part is variable operation and maintenance cost.

\section{Maximizing the reliability}

The reliability objective criterion of maximizing the average value of reliability index (RI) in the planning period is considered. The reliability index in every sub-period is nothing but the ratio of net reserve in that sub-period to gross reserve in that sub-period. The net reserve can be determined as the difference between the total available generation, capacity of generators that are under maintenance and load demand. The gross reserve can be obtained by taking difference between the total available generation and load demand [23]. The reliability objective function is stated as

$\boldsymbol{F}_{2}=\operatorname{Max} \frac{1}{T} \sum_{t=1}^{T} \boldsymbol{R I}(\boldsymbol{t})$

where

$$
\boldsymbol{R I}(\boldsymbol{t})=\frac{\sum_{i=1}^{N} \boldsymbol{P}_{i}^{\max }-\sum_{i=1}^{N} \boldsymbol{P}_{i}^{\max } \boldsymbol{U}_{i t}-\boldsymbol{D}_{\boldsymbol{t}}}{\sum_{i=1}^{N} \boldsymbol{P}_{i}^{\max }-\boldsymbol{D}_{\boldsymbol{t}}}
$$

\subsection{Equality and Inequality constraints}

The best compromise solution obtained for the multi objective GMS must satisfy the following set of equality and inequality constraints.

\subsubsection{Maintenance Window Constraint}

The planned preventive maintenance work of each power generating unit present in the system must be scheduled and carried out between its earliest and latest period allowed for maintenance. This constraint ensures that once maintenance of the unit $i$ is initiated, the work have to be continued without any break off for the time period that is exactly equal to maintenance duration of unit $i$. The constraint can be expressed as

$$
U_{i t}=\left\{\begin{array}{rr}
1, & t=S_{i}, \ldots, S_{i}+M_{i}-1 \\
0, & \text { otherwise }
\end{array}\right.
$$

\subsubsection{Crew Constraint}

It depends on the availability of crew for maintenance work. It guarantees that no two units can be maintained by the same maintenance crews. It is expressed in terms of $U_{i t}$ variables of the second unit $i 2$ as follows

$$
\sum_{t=S_{i 1}}^{S_{i 1}+M_{i 1}-1} \boldsymbol{U}_{i 2, t}=0
$$

Equation (5), states that unit ' 2 ' should not undergo any maintenance work when the duration on which unit ' 1 ' is under maintenance.

\subsubsection{Priority Constraint}

In some occasions, some of the generating units need maintenance to be completed first before the starting of maintenance of other generating units. This is attained with the help of priority constraint. This constraint specifies the priority in which maintenance on the generators has to be carried out. For example, if maintenance of unit ' 1 ' is to be completed before the starting of maintenance of unit ' 2 ', then this constraint is given by

$$
S_{2}>S_{1}+M_{1}-1
$$




\subsubsection{Resource Constraint}

This constraint guarantees that the capacity on maintenance is less than the gross reserve at any time period in the planning horizon.

$\sum_{i=1}^{N} \boldsymbol{R}_{i} \boldsymbol{U}_{i t} \leq\left(\sum_{i=1}^{N} \boldsymbol{P}_{i}^{\max }-\boldsymbol{D}_{\boldsymbol{t}}\right)$

In this paper, it is assumed that the rating of unit $i$ is exactly equal to the maximum power that can be generated by unit $i$.

\subsubsection{Generator Limit Constraint}

The active power output from every power generating units must be within their lower and upper bounds. This is given by

$$
\boldsymbol{P}_{i}^{\min } \leq \boldsymbol{P}_{i t} \leq \boldsymbol{P}_{i}^{\max }
$$

\subsubsection{Power Balance Constraint}

This constraint ensures that the total generation of all the units in every sub-period must be equal to the load demand on that sub-period t. This is given by $\sum_{i=1}^{N} \boldsymbol{P}_{i t}=\boldsymbol{D}_{t}$

\section{Proposed Solution Methodology}

In this paper, GMS is formulated as a mixed integer problem. By including rounding off operator, DE is modified to handle integer variables that denotes the starting period for maintenance of generators. Lagrange multiplier approach that supports to economically dispatch the available generation is embedded into DE searching routine which helps $\mathrm{DE}$ in finding the faithful starting period for maintenance with minimum production cost. When more than one objective function is to be optimized in unison, it is necessary to make a compromise between those objectives to get a best solution. Fuzzy set theory has also been employed into DE search process in order to extract efficiently the best compromise non inferior solution. In fuzzy set theory, each objective is defined by membership functions. The membership function signifies the degree of satisfaction of the objective. If the value of the membership function is 1 , the solution is said to be fully satisfied. For an unsatisfied solution, its value becomes zero [24]. The two objectives described in the previous text are fuzzified as follows

\subsection{Fuzzy model for cost objective}

In the context of GMS, the cost objective is to be minimized. For the cost objective, the fuzzy membership function is symbolized as $\mu_{\mathrm{F} 1}$. As discussed earlier, for this objective, if the cost is equal to or nearer to its minimum value, the value of $\mu_{\mathrm{F} 1}$ is unity, on the other hand, if the cost is equal to or nearer to maximum value, the value of $\mu_{\mathrm{F} 1}$ is zero otherwise the membership function value $\mu_{\mathrm{F} 1}$ is calculated as follows.

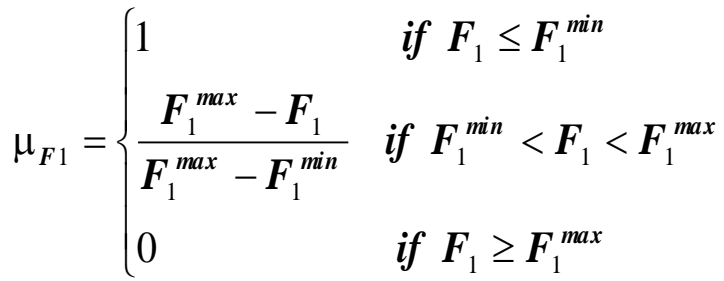

Thus the membership function for the objective of minimizing the overall operation cost is monotonically decreasing membership function.

\subsection{Fuzzy model for reliability objective}

In the GMS framework, the reliability needs to be maximized in order to increase the system security. For this objective, the fuzzy membership function is symbolized as $\mu_{\mathrm{F} 2}$. For the objective of maximizing average value of reliability index, if RI is equal to or nearer to its minimum value, the value of $\mu_{\mathrm{F} 2}$ is zero, on the other hand, if the value of RI is equal to or nearer to maximum value, the value of $\mu_{\mathrm{F} 2}$ approaches unity otherwise the membership function value $\mu_{\mathrm{F} 2}$ is obtained as follows.

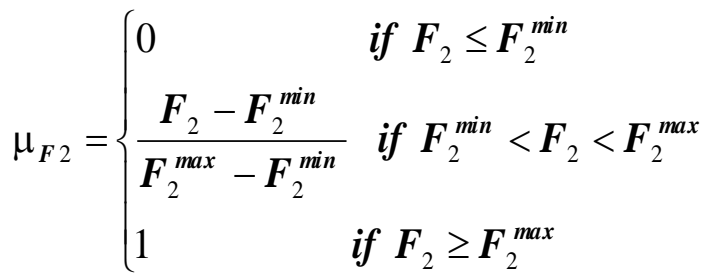

Hence the membership function for the objective of minimizing the overall operation cost is monotonically increasing membership function.

The proposed fuzzy clustered multi objective hybrid differential evolution algorithm for solving GMS has the following steps. 


\subsection{Initialization}

In DE, using the rounding off operator, the population of parent or target vector of integers is randomly initialized $(\mathrm{X}$ ) between its corresponding earliest and latest starting period that specifies the starting period of each generator present in the system.

$$
\left(X_{i}^{k}\right)=\operatorname{round}\left(E_{i}^{k}+\operatorname{random}(0,1) \cdot\left(L_{i}^{k}-E_{i}^{k}\right)\right)
$$

where $\mathrm{k}=1,2, \ldots$, number of populations (NP). If the integer variables thus selected violates crew or priority constraint, reinitialize the corresponding integer randomly in order to satisfy those constraints. The integer variables are checked for convincing maintenance window constraint. After satisfying constraints (4), (5), (6) and (7), the status of each unit $i$ is fixed at ' 1 ' from its corresponding starting period up to its respective maintenance duration week in order to indicate that unit $\mathrm{i}$ is put out for maintenance and ' 0 ' during other periods. Using Lagrange Multiplier method, the committed generators are economically dispatched to meet the weekly load demand with minimum overall operation cost (1). The Lagrange Multiplier method has the following steps.

Step 1: Starting with an initial value of $\lambda$, power output of each committed generator $\left(\mathrm{P}_{\mathrm{i}}\right)$ in sub period $t$ is obtained using

$\boldsymbol{P}_{i}=\frac{\lambda-\boldsymbol{b}_{i}}{2 \boldsymbol{c}_{i}}$

The output power $\left(\mathrm{P}_{\mathrm{i}}\right)$ from each generator is checked whether it exceeds lower or upper bounds. If it violates lower bound, it is fixed at its minimum limit or if it exceeds the upper bound, it is set at its maximum limit.

Step 2: The change in output power is calculated using

$$
\Delta \boldsymbol{P}^{K}=\boldsymbol{D}_{t}-\sum_{i=1}^{N C G} \boldsymbol{P}_{i}
$$

where NCG stands for number of committed generators.

Step 3: The new value of $\lambda$ for successive iteration is obtained by adding change in $\lambda$ with previous value of $\lambda$ as follows

$$
\begin{aligned}
\Delta \lambda^{K} & =\frac{\Delta \boldsymbol{P}^{K}}{\sum_{i=1}^{N C G} \frac{1}{2 c_{i}}} \\
\lambda^{K+1} & =\lambda^{K}+\Delta \lambda^{K}
\end{aligned}
$$

The steps 1 through 3 are repeated until $\Delta \mathrm{P}$ becomes zero. Hence equations (8) and (9) are satisfied.

The average value of reliability index is calculated using equation (2). Then for each objective, the membership function is calculated using equations (10) and (11). For each vector $\mathrm{k}$ in population, the normalized membership function value is calculated using

$$
\mu^{k}=\frac{\sum_{j=1}^{2} \mu_{F j}^{k}}{\sum_{k=1}^{N P} \sum_{j=1}^{2} \mu_{F j}^{k}}
$$

where $\mathrm{j}$ stands for objective index. The vector for which the normalized membership function value $\mu^{k}$ attains maximum can be chosen as a best compromise non dominated solution for GMS. Thus equation (17) is considered as the fitness function to be maximized.

\subsection{Mutation}

The main operator of DE is mutation. Mutation means making sudden change with a random element. The mutant or donor vector is created in the mutation operation. The mutation strategy that is used in this work is $\mathrm{DE} / \mathrm{rand} / 1$ in which three distinct vectors $\mathrm{r} 1, \mathrm{r} 2$ and $\mathrm{r} 3$ are randomly chosen from $[1, \mathrm{NP}]$ that is also different from base index $\mathrm{k}$. The donor integer vector for each target vector is created by adding scaled difference between any two vectors with third vector. The scaling factor $F$ lies between $[0.1,1]$. The donor vector is created as follows

$\left(U_{k}^{G+1}\right)=\left(X_{r 1}^{G}+\operatorname{round}\left(F \cdot\left(X_{r 2}^{G}-X_{r 3}^{G}\right)\right)\right)$

The donor integer vector thus created is checked for crew and priority constraint. If any of the constraint is violated for a particular generator, the corresponding integer variable is selected randomly between its earliest and latest starting period until 
the crew and priority constraints are satisfied and then the integer variables are checked in order to satisfy constraints (6) and (7).

\subsection{Crossover}

The trial integer vector is created in this phase with the help of binomial crossover operator. The individual gene for trial vector are imitated from corresponding gene of donor vector if a random number is lesser than or equal to crossover factor (CR) otherwise imitated from donor vector as follows

$V_{h k}^{G+1}=\left\{\begin{array}{l}U_{h k}^{G+1}, \text { random }_{k}(0,1) \leq \boldsymbol{C R} \\ \boldsymbol{X}_{h k}^{G}, \text { otherwise }\end{array}\right.$

where $\mathrm{h}=1,2, \ldots$, number of integers $(\mathrm{NI})$ and crossover factor $\mathrm{CR} \in[0,1]$. The status of each unit $i$ is set at ' 1 ' in order to indicate that unit $i$ is taken out for maintenance and ' 0 ' during other periods. Using equations (13), (14), (15) and (16), the available generation is optimally dispatched with minimum production cost and hence generator limit and power balance constraints are satisfied. The average value of reliability index is calculated using (2). The membership function value for cost and reliability objective is calculated using (10) and (11) and the normalized membership function value for every individual population $\mathrm{k}$, is calculated using (17). The vector for which the normalized membership function value $\mu^{\mathrm{k}}$ attains maximum can be chosen as a best compromise non dominated solution.

\subsection{Selection}

The population of target integer vector for the next generation is created in this phase using equation (17) as follows

$X_{k}^{G+1}= \begin{cases}V_{k}^{G+1}, & \text { if } \mu^{G+1}>\mu^{G} \\ X_{k}^{G}, & \text { otherwise }\end{cases}$

The population for next generation is copied from trial vector if it has better normalized membership function value otherwise target vector is copied. The above steps are repeated until maximum number of generations.

\section{Simulation Results and Discussion}

The solution of a small size system of having 4 generating units and real size system of having 22 units [5] using proposed FC-MOHDE are presented. In the proposed approach, Lagrange Multiplier approach is included into the DE procedure with the intention of optimally dispatch the committed generating units to meet the weekly power demand with minimum production cost. To achieve the best compromise non dominated solution for GMS from the non dominated solution set that is obtained with the help of MOHDE; fuzzy set theory is also incorporated into the algorithm of the MOHDE. In this work, GMS is modeled as a MOP subject to various system and operational constraints. The program is developed on a MATLAB platform. To examine the performance of the proposed approach, two case studies are discussed below.

\subsection{Case 1: 4 Units System}

A small size system having 4 generators that has to be maintained over a planning period of 8 weeks. All the 4 units have to be maintained only once during the planning period. Due to priority constraint, the preventive maintenance of unit 1 must be finished before the initiation of maintenance of unit 2. Similarly, due to man power constraint, unit1 and unit 2 should not be taken off for maintenance during same period. The generator data of 4 units system and the weekly load profile are given in [25].

The minimum and maximum values of individual objectives are obtained by giving full weightage to one objective and neglecting the other. In this way, the extreme values of cost and reliability index are obtained. When the objective overall operation cost is minimized, then if the reliability index is computed, it is also minimum. When the objective of average value of reliability index is maximized, then the corresponding overall operation cost is also maximum. Thus if the system security is increased, the expenditure also gets increased. The extreme values of cost and average value of reliability index for 4 units system satisfying all above mentioned constraints are given table 1 .

Table 1: Extreme values of individual objectives for 4 units system

\begin{tabular}{c|c|c}
\hline $\begin{array}{c}\text { Objective } \\
\text { function }\end{array}$ & $\begin{array}{c}\text { Minimum } \\
\text { value }\end{array}$ & $\begin{array}{c}\text { Maximum } \\
\text { value [25] }\end{array}$ \\
\hline
\end{tabular}




\begin{tabular}{l|c|c}
\hline F1 & $\$ 3391993.59$ & $\$ 3400434.56$ \\
\hline F2 & 0.5525 & 0.5588 \\
\hline
\end{tabular}

For the objective of minimizing overall operation cost, the maintenance of 4 generating units $1,2,3$ and 4 begins in the period (week) 3, 7, 1 and 7 respectively. For the goal of maximizing the average value of reliability index, maintenance of 1 through 4 units starts during 1, 6, 7 and 7 respectively [25].

The best compromise non dominated solution found using proposed FC-MOHDE algorithm is shown in table 2. Since the starting period is taken as decision variable, the number of variables for 4 units system is 4 . The control parameters of the algorithm that is population size, maximum number of generation, mutation rate and crossover rate are set at 25, 500, 0.7 and 0.9 respectively.

For the best compromise solution obtained, the maintenance schedule is shown in table 3 .

Table 2: Best compromise solution for 4 units system

\begin{tabular}{c|c}
\hline \multicolumn{2}{|c}{ system } \\
\hline Cost (\$) & $\begin{array}{c}\text { Average value of } \\
\text { RI }\end{array}$ \\
\hline
\end{tabular}

\begin{tabular}{l|l}
\hline 3396397.47 & 0.5567 \\
\hline
\end{tabular}

Table 3: Maintenance schedule of 4 units system

\begin{tabular}{l|l|l|l|l|l|l|l|l}
\hline Unit/Week & $\mathbf{1}$ & $\mathbf{2}$ & $\mathbf{3}$ & $\mathbf{4}$ & $\mathbf{5}$ & $\mathbf{6}$ & $\mathbf{7}$ & $\mathbf{8}$ \\
\hline $\mathbf{1}$ & $\mathbf{1}$ & $\mathbf{1}$ & $\mathbf{1}$ & $\mathbf{1}$ & 0 & 0 & 0 & 0 \\
\hline $\mathbf{2}$ & 0 & 0 & 0 & 0 & 0 & $\mathbf{1}$ & $\mathbf{1}$ & 0 \\
\hline $\mathbf{3}$ & 0 & 0 & 0 & 0 & 0 & 0 & $\mathbf{1}$ & $\mathbf{1}$ \\
\hline $\mathbf{4}$ & $\mathbf{1}$ & 0 & 0 & 0 & 0 & 0 & 0 & 0 \\
\hline
\end{tabular}

In table 3 , it can seen that maintenance of units 1 through 4 begins in first, sixth, seventh and first week respectively and are under maintenance state for the duration equal to its respective maintenance duration weeks satisfying maintenance window constraints. From table 3, it is clear that unit 1 and 2 are not taken off for preventive maintenance at the same time thereby satisfying crew constraint. In the same way, maintenance of unit 2 gets started only after the finishing of maintenance of unit 1, thereby satisfying priority constraint. The economic dispatch of the available generation from the committed generating units to meet the load demand is shown in table 4. In table 4, it can be seen that, the sum of the output power on every week is exactly equal to weekly load demand, thereby satisfying power balance constraint. In every sub-period, the capacity on maintenance is less than the gross reserve thereby satisfying resource constraint as shown in figure 1 . The minimum and maximum reserve that can be obtained is $103 \mathrm{MW}$ in 7th week and $534 \mathrm{MW}$ in 5th week as shown in figure 2 .

Table 4: Generation schedule of 4 units system

\begin{tabular}{c|c|c|c|c|c|c|c|c}
\hline Unit/Week & $\mathbf{1}$ & $\mathbf{2}$ & $\mathbf{3}$ & $\mathbf{4}$ & $\mathbf{5}$ & $\mathbf{6}$ & $\mathbf{7}$ & $\mathbf{8}$ \\
\hline $\mathbf{1}$ & $\mathbf{0}$ & $\mathbf{0}$ & $\mathbf{0}$ & $\mathbf{0}$ & 73.0 & 125.2 & 137.9 & 118.7 \\
\hline $\mathbf{2}$ & 127.6 & 125.1 & 129.1 & 130.2 & 94.5 & $\mathbf{0}$ & $\mathbf{0}$ & 142.3 \\
\hline $\mathbf{3}$ & 121.4 & 118.9 & 122.9 & 124.0 & 88.5 & 142.7 & $\mathbf{0}$ & $\mathbf{0}$ \\
\hline $\mathbf{4}$ & $\mathbf{0}$ & 21.0 & 24.0 & 24.8 & 0 & 39.1 & 49.1 & 34.0 \\
\hline
\end{tabular}




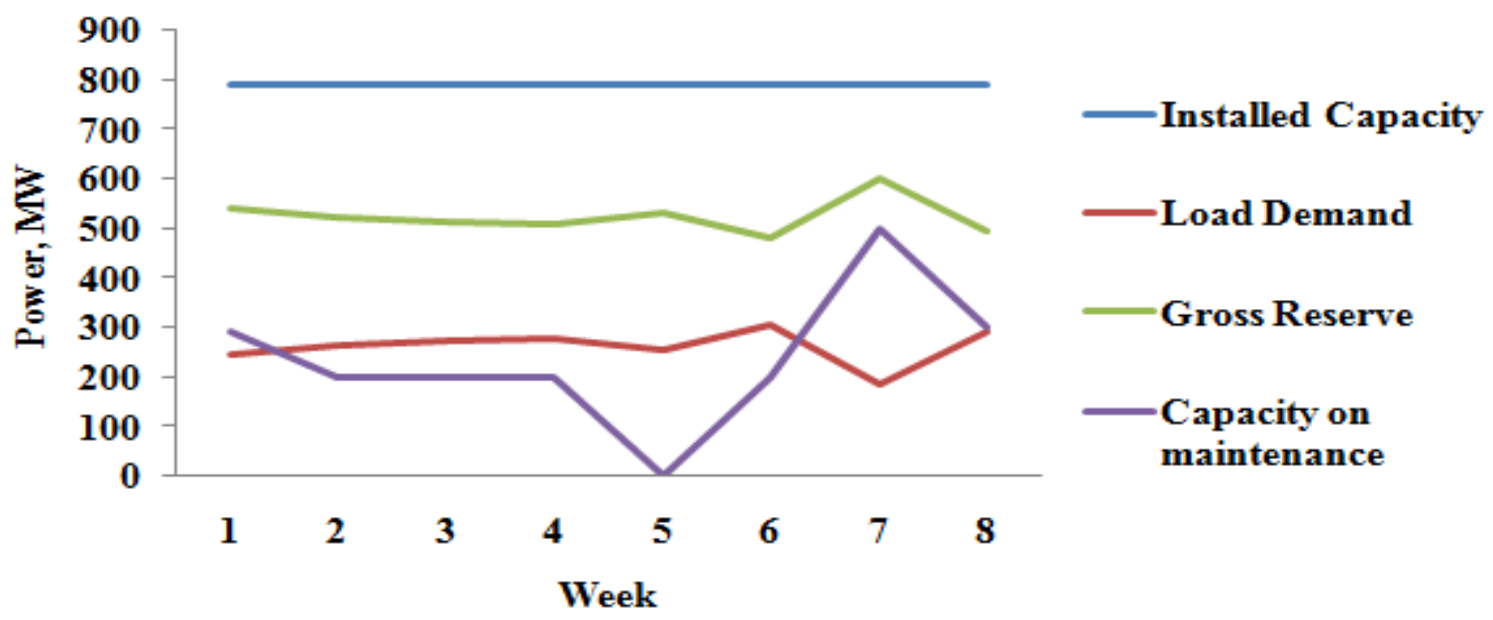

Figure 1: Capacity on Maintenance for 4 units system

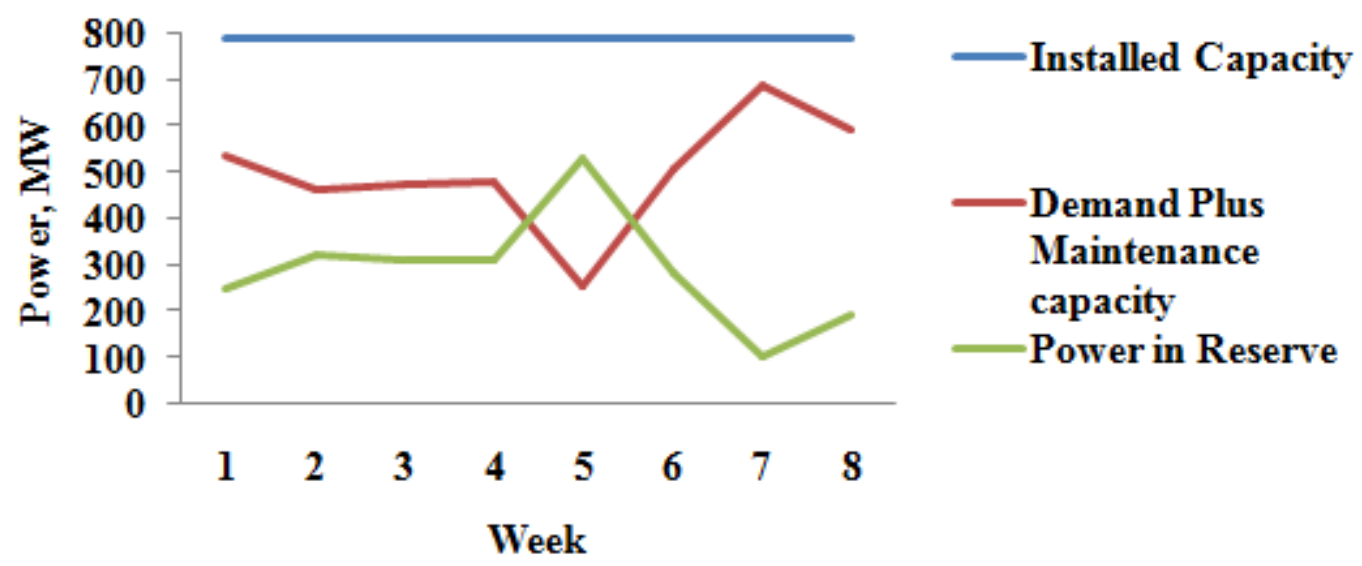

Figure 2: Reserve profile for 4 units system

\subsection{Case 2: 22 Units System}

A real size system having 22 generating units that has to be maintained over a planning horizon of one year divided into a sub period of one week. In the prescribed planning horizon, each and every generator must be taken out for maintenance only once. The details of the power production units are given in [25].

The priority constraint restricts the starting of maintenance of unit 3 and 6 in the sense that its repair works can be carried out only after the completion of maintenance of unit 2 and 5 respectively. Likewise, crew constraint limits the simultaneous maintenance of units 15 and 16 , units 21 and 22. The weekly load profile is given in [25].

The minimum and maximum values of cost and average value of reliability index obtained by optimizing these factors individually for 22 units system satisfying all the constraints yields the following results as shown in table 5 .

\section{Table 5: Extreme values of individual} objectives for 22 units system 


\begin{tabular}{c|c|c}
\hline $\begin{array}{c}\text { Objective } \\
\text { function }\end{array}$ & Minimum value & $\begin{array}{c}\text { Maximum } \\
\text { value [25] }\end{array}$ \\
\hline $\mathrm{F}_{1}$ & $\$ 148580466.97$ & $\$ 148624224.65$ \\
\hline $\mathrm{F}_{2}$ & 0.8179 & 0.8235 \\
\hline
\end{tabular}

The beginning of units' maintenance for the objective of minimizing the overall operation cost and maximizing average value of reliability index is shown in table 6 .

The best compromise non dominated solution found for 22 units system using proposed FC-MOHDE algorithm is shown in table 7. Since Lagrange Multiplier method is used for optimally dispatch the available generation with minimum production cost, the power balance constraint have been proved. Since the starting period for maintenance of power production units is taken as decision variable, the number of variables for 22 units system is 22. The tuning parameters of the algorithm that is population size, maximum number of generation, mutation rate and crossover rate are set at $75,2000,0.5$ and 0.94 respectively. The corresponding maintenance schedule is shown in table 8 .

Form table 8 , it is clear that each unit is put out for maintenance only once during the planning horizon. Also the units are under maintenance state continuously without any interruption for the duration equal to its respective maintenance duration weeks satisfying maintenance window constraints. Table 8 clearly indicates that the maintenance activities of units 15 and 16, units 21 and 22 are not done simultaneously, thereby satisfying crew constraint. Likewise, it can be seen that the maintenance of units 2 and 5 come to an end before the starting of maintenance of units 3 and 6 respectively, thereby upholding priority constraint. The capacity on maintenance and gross reserve in every sub-period is shown in figure 3. From the figure, it is evident that the capacity of units under maintenance is less than the gross reserve such that resource constraint gets satisfied.
Table 6: Maintenance Schedule

\begin{tabular}{|c|c|c|}
\hline $\begin{array}{l}\text { Unit } \\
\text { No. }\end{array}$ & F1 & F2 [25] \\
\hline 1 & 1 & 7 \\
\hline 2 & 32 & 14 \\
\hline 3 & 46 & 17 \\
\hline 4 & 12 & 4 \\
\hline 5 & 1 & 10 \\
\hline 6 & 46 & 21 \\
\hline 7 & 32 & 5 \\
\hline 8 & 14 & 17 \\
\hline 9 & 41 & 41 \\
\hline 10 & 7 & 7 \\
\hline 11 & 1 & 1 \\
\hline 12 & 16 & 7 \\
\hline 13 & 20 & 15 \\
\hline 14 & 1 & 8 \\
\hline 15 & 1 & 10 \\
\hline 16 & 12 & 15 \\
\hline 17 & 19 & 13 \\
\hline 18 & 16 & 8 \\
\hline 19 & 26 & 16 \\
\hline 20 & 4 & 14 \\
\hline 21 & 15 & 7 \\
\hline 22 & 19 & 13 \\
\hline
\end{tabular}

Table 7: Best compromise solution for 22 units system

\begin{tabular}{c|c}
\hline Cost (\$) & $\begin{array}{c}\text { Average value of Reliability } \\
\text { Index }\end{array}$ \\
\hline 148617117.5 & 0.8215 \\
\hline
\end{tabular}

Table 8: Maintenance Schedule for best compromise solution

\begin{tabular}{|l|l|l|l|l|l|}
\hline Week & Maintenance & Units under maintenance & Week & Maintenance & Units under maintenance \\
\hline
\end{tabular}

International Journal of Intelligent Engineering and Systems, Vol.9, No.1, 2016 


\begin{tabular}{|c|c|c|c|c|c|}
\hline & Capacity & & & Capacity & \\
\hline 1 & 0 & $* * * * *$ & 27 & 100 & 14 \\
\hline 2 & 0 & $* * * * *$ & 28 & 100 & 14 \\
\hline 3 & 0 & $* * * * *$ & 29 & 0 & $* * * * *$ \\
\hline 4 & 320 & 4,15 & 30 & 0 & $* * * * *$ \\
\hline 5 & 420 & $4,15,17$ & 31 & 0 & $* * * * *$ \\
\hline 6 & 520 & $1,4,15,17$ & 32 & 0 & $* * * * *$ \\
\hline 7 & 420 & $1,15,17$ & 33 & 0 & $* * * * *$ \\
\hline 8 & 1130 & $1,10,12,15,17$ & 34 & 0 & $* * * * *$ \\
\hline 9 & 1350 & $1,2,10,12,17,18,21$ & 35 & 0 & $* * * * *$ \\
\hline 10 & 1250 & $1,2,10,12,18,21$ & 36 & 0 & $* * * * *$ \\
\hline 11 & 1250 & $1,2,10,12,18,21$ & 37 & 0 & $* * * * *$ \\
\hline 12 & 1030 & $10,12,16,18$ & 38 & 0 & $* * * * *$ \\
\hline 13 & 1370 & $3,10,12,16,18,22$ & 39 & 0 & $* * * * *$ \\
\hline 14 & 1490 & $3,10,12,16,20,22$ & 40 & 0 & $* * * * *$ \\
\hline 15 & 1671 & $3,5,10,11,12,16,20,22$ & 41 & 650 & 9 \\
\hline 16 & 1691 & $5,10,11,16,19,20,22$ & 42 & 650 & 9 \\
\hline 17 & 1566 & $5,7,10,11,16,19,22$ & 43 & 650 & 9 \\
\hline 18 & 1106 & $5,7,10,11,19$ & 44 & 650 & 9 \\
\hline 19 & 795 & $5,7,10$ & 45 & 740 & 6,9 \\
\hline 20 & 90 & 5 & 46 & 90 & 6 \\
\hline 21 & 100 & 8 & 47 & 90 & 6 \\
\hline 22 & 100 & 8 & 48 & 90 & 6 \\
\hline 23 & 200 & 8,14 & 49 & 0 & $* * * * *$ \\
\hline 24 & 300 & $8,13,14$ & 50 & 0 & $* * * * *$ \\
\hline 25 & 200 & 13,14 & 51 & 0 & $* * * * *$ \\
\hline 26 & 200 & 13,14 & 52 & 0 & $* * * * *$ \\
\hline
\end{tabular}

The available reserve power can be calculated by taking difference between installed capacity, demand and capacity on maintenance. The lowest and highest values of reserve that can be obtained for 22 units system is 872 MW and 2292 MW respectively as shown in figure 4 . 


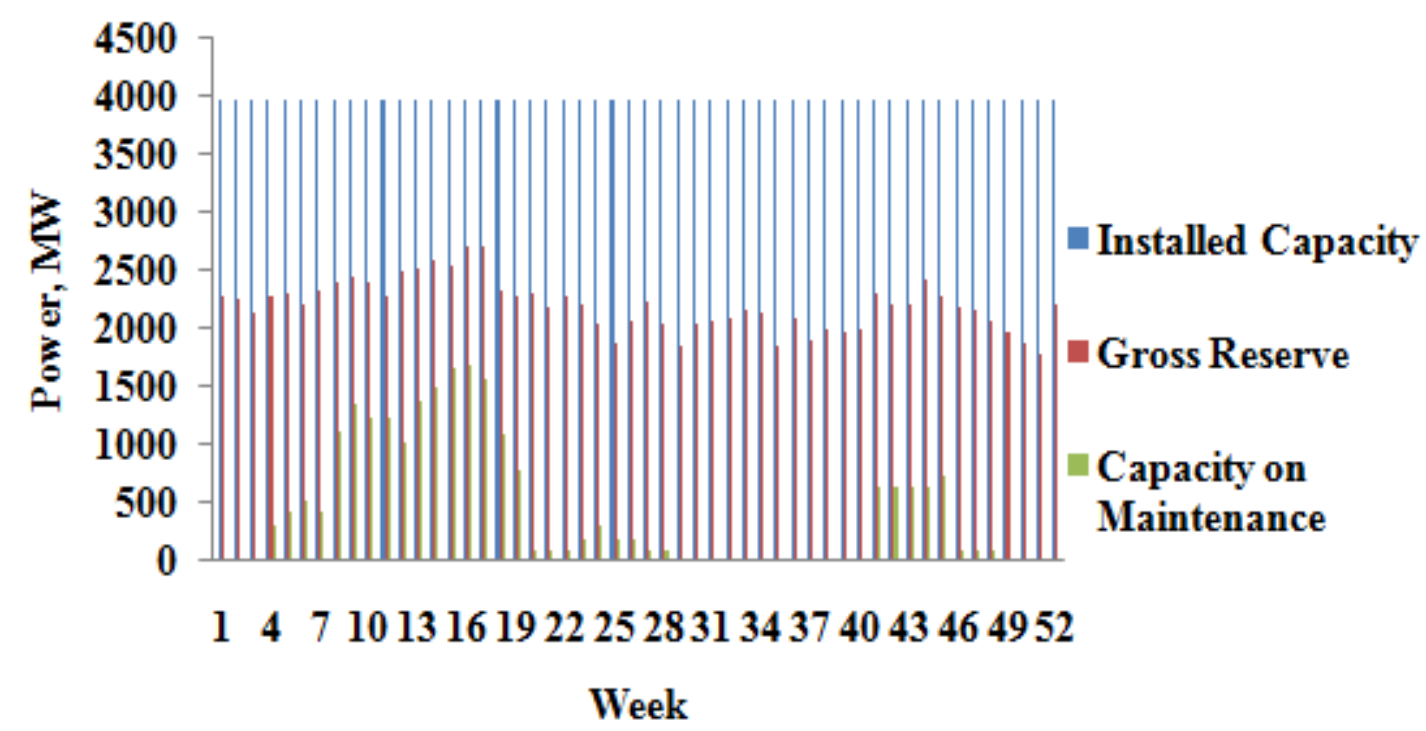

Figure 3: Capacity on maintenance

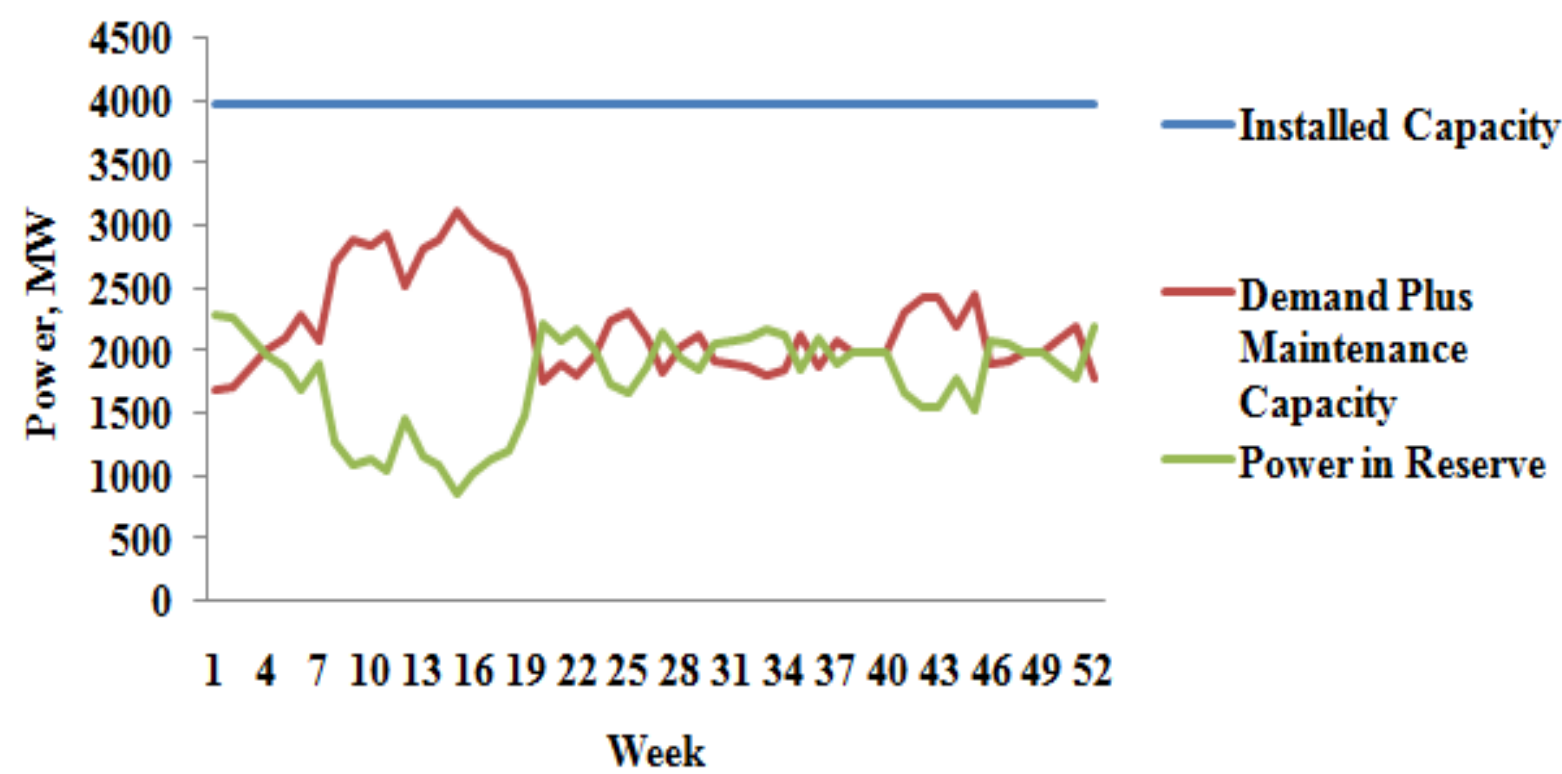

Figure 4: Reserve profile for 22 units system

\section{Conclusion}

In this paper, the problem of scheduling of power generating units for maintenance has been formulated as a multi objective problem with cost and reliability as objectives. The problem is subjected to various set of hard and soft constraints. Fuzzy clustered multi objective differential evolution algorithm has been proposed to solve multi objective GMS. In the proposed approach, integer coded differential evolution algorithm acts as a main optimizer for which Lagrange Multiplier method helps in finding minimum production cost maintenance schedule. The fuzzy set theory helps in finding best compromise non dominated solution for GMS. The proposed approach is validated by considering a small and real size system. The test results reveal the capability of the algorithm in providing best compromise solution between cost and reliability. The future scope of this work is that the transmission maintenance 
scheduling problem can be added as a constraint to find maintenance schedule for GMS problem.

\section{Acknowledgment}

The authors gratefully acknowledge the support and facilities provided by the authorities of Annamalai University, Annamalainagar, India to carry out this research work.

\section{Nomenclature}

$\mathrm{t}$ - $\quad$ Time period index (week)

$\mathrm{T}$ - Total number of sub periods (weeks) in the planning horizon

$\mathrm{i}$ - $\quad$ Power generating unit index

$\mathrm{N}$ - $\quad$ Total number of generators

$\mathrm{H}$ - Number of hours in a sub-period (week)

$a_{i}, b_{i}, c_{i}-$ Fuel cost coefficients

$v_{i}-\quad$ Variable operation and maintenance cost of unit $i$, \$/MWh

$\mathrm{U}_{\mathrm{it}}$ - $\quad$ State variable equal to 1 if the unit $i$ in sub period $t$ is under maintenance and 0 otherwise

$\mathrm{R}_{\mathrm{i}}$ - $\quad$ Rating of unit $i$, MW

$\mathrm{P}_{\text {it }}$ - Power output from generating unit $i$ in sub-period $t$, MW

$\mathrm{RI}(t)$ - Reliability index in sub-period $t$

$\mathrm{P}_{\mathrm{i}}^{\text {min }}$ - Minimum limit generating unit $i$

$\mathrm{P}_{\mathrm{i}}{ }^{\max }$ - Maximum limit generating unit $i$

$D_{\mathrm{t}}$ - $\quad$ Active power load demand in sub-period $t, \mathrm{MW}$

$\mathrm{E}_{\mathrm{i}}$ - $\quad$ Earliest period in which maintenance of unit $i$ can start

$\mathrm{L}_{\mathrm{i}}$ - $\quad$ Latest period in which maintenance of unit $i$ can start

$\mathrm{S}_{\mathrm{i}}$ - $\quad$ Starting period of maintenance of unit $i \in\left[L_{i}, E_{i}\right]$

$\mathrm{M}_{\mathrm{i}}$ - $\quad$ Maintenance duration of unit $i$

\section{References}

[1] K.Y. Huang and H.T. Yang, "Effective algorithm for handling constraints in generator maintenance scheduling", IEE Proceedings on Generation, Transmission and Distribution, Vol. 149, No. 3, pp. 274-282, 2002.

[2] J. Yellen, T.M. Al-Khamis, S. Vermuri and L.
Lemonidis, "A decomposition approach to unit maintenance scheduling", IEEE Transactions on Power Systems, Vol. 7, No. 2, pp. 726-733, 1992.

[3] T. Satoh and K. Nara, "Maintenance Scheduling by using Simulated Annealing Method", IEEE Transactions on Power Systems, Vol. 6, No. 5, pp. 850 - 857, 1991.

[4] S. Baskar, P. Subbaraj, M.V.C. Rao and S. Tamilselvi, "Genetic algorithms solution to generator maintenance scheduling with modified genetic operators", IEE Proceedings on Generation, Transmission, Distribution, Vol. 150, No. 1, pp. 56 60, 2003.

[5] I. El - Amin, S. Duffuaa and M. Abbas, "A Tabu search algorithm for maintenance scheduling of generating units", Electric Power System Research, Vol. 54, pp. 91 - 99, 2000.

[6] E. K. Burke and A.J. Smith, "Hybrid Evolutionary Techniques for the Maintenance Scheduling Problem", IEEE Transactions on Power Systems, Vol. 15, No. 1, pp. 122 - 128, 2000.

[7] M.Y. El-Sharkh, A.A. El-Keib and H. Chen, "A fuzzy evolutionary programming based solution methodology for security-constrained generation maintenance scheduling”, Electric Power System Research, Vol. 67, pp. 67 - 72, 2003.

[8] C.A. Koay and D. Srinivasan, "Particle swarm optimization-based approach for generator maintenance scheduling", Proc. of IEEE Swarm Intelligence Symposium, pp. 167-173, 2003.

[9] K.P. Dahal, C.J. Aldridge and J.R. McDonald, "Generator maintenance scheduling using a genetic algorithm with a fuzzy evaluation function", Fuzzy Sets and System, Vol. 102, pp. 21 - 29, 1999.

[10] W.K. Foong, H.R. Maier and A.R. Simpson, "Power plant maintenance scheduling using ant colony optimization: an improved formulation", Engineering Optimization, Vol. 40, No. 4, pp 309-329, 2008.

[11] Y. Wang and E. Handschin, "A new genetic algorithm for preventive unit maintenance scheduling of power systems", Electrical Power and Energy Systems, Vol. 22, pp. 343 - 348, 2000.

[12] A. Volkanovski, B. Mavko, T. Bosevski, A. 
Causevski and M. Cepin, "Genetic algorithm optimization of the maintenance scheduling of generating units in a power system", Reliability Engineering and System Safety, Vol. 93, pp. 757 767, 2008.

[13] J.P. Stremel, "Maintenance Scheduling For Generation System Planning”, IEEE Transactions on Power Apparatus and Systems, Vol. 100, No. 3, pp.1410 - 1419, 1981.

[14] A.V. Fetanat and G. Shafipour, "Harmony Search Algorithm Based 0-1 Integer Programming for Generation Maintenance Scheduling in Power Systems", Journal of Theoretical and Applied Information Technology, Vol. 24, No. 1, pp. 1 - 10, 2011.

[15] S.C. Satapathy, A. Naik and K. Parvathi, "0-1 integer programming for generation maintenance scheduling in power systems based on teaching learning based optimization (TLBO)", Communications in Computer and Information Science (CCIS) series, Vol. 306, No. 2, pp. 53-63, 2012.

[16] H. Kim, Y. Hayashi and K. Nara, “An algorithm for Thermal Unit Maintenance Scheduling through combined use of GA, SA and TS", IEEE Transactions on Power Systems, Vol. 12, No. 1, pp. 329-335, 1997.

[17] R.C. Leou, "A new method for unit maintenance scheduling considering reliability and operation expense", Electrical Power and Energy Systems, Vol. 28, No. 7, pp. 471-481, 2006.

[18] B. Kralj and R. Petrovic, "A multi objective optimization approach to thermal generating units maintenance scheduling", European Journal of Operational Research, Vol. 84, pp. 481-493, 1995.

[19] B. Kralj and N. Rajakovic, "Multi-objective programming in power system optimization: new approach to generator maintenance scheduling", Electrical Power and Energy Systems, Vol. 16, No. 4, pp. 211 - 220, 1994.

[20] R. Storn and K.V. Price, "Differential evolution - A simple and efficient heuristic for global optimization over continuous spaces", Journal of Global Optimization, Vol. 11, pp. 341 - 359, 1997.
[21] R. Storn and K.V. Price, "Minimizing the real function of the ICEC ${ }^{\text {cee }} 96$ contest by differential evolution", Proceedings of IEEE Conference on Evolutionary Computation, pp. 842-844, Nagoya, Japan, 1996.

[22] S. Das, A. Abraham, U.K. Chakraborty and A. Konar, "Differential Evolution Using a Neighborho od-Based Mutation Operator", IEEE Transactions o n Evol-utionary Computation, Vol. 13, No. 3, pp. $526-553,2009$.

[23] A.J. Conejo, R.G. Bertrand and M.D. Salazar, "Generation Maintenance Scheduling in Restructured Power Systems", IEEE Transactions on Power Systems, Vol. 20, No. 2, pp. 984 - 992, 2005.

[24] J.S. Dhillon, S.C. Parti and D. P. Kothari, "Stochastic economic emission load dispatch", Electric Power System Research, Vol. 26, pp. 179 186, 1993.

[25] G. Balaji, R. Balamurugan and L. Lakshminarasimman, "Reliability based Generator Maintenance Scheduling using Integer Coded Differential Evolution Algorithm", International Journal of Computer Applications, Vol. 131, No.6, pp. $27-38,2015$. 